\title{
TEXTURE CLASSIFICATION BASED ON OVERLAPPED TEXTON CO-OCCURRENCE MATRIX (OTCOM) FEATURES
}

\author{
Patnala S. R. Chandra Murthy ${ }^{1}$, U Ravi Babu' ${ }^{2}$ R Venkatalakshmi ${ }^{3}$ \\ ${ }^{1}$ Assistant Professor, Department of CSE, University College of Engineering and Technology, ANU-Guntur \\ ${ }^{2}$ Professors, Department of CSE, Malla Reddy Engg, College (Autonomous) TS, INODA \\ ${ }^{3}$ Research Schalor, JNTUK. Kakinada, India
}

\begin{abstract}
Abstract: The pattern identification problems such as stone, rock categorization and wood recognition are used texture classification technique due to its valuable usage in it. Generally, texture analysis can be done one of the two ways i.e. statistical and structural approaches. More problems are occurred when working with statistical approaches in texture analysis for texture categorization. One of the most popular statistical approaches is Gray Level Co-occurrence Matrices (GLCM) approach. This approach is used to discriminating different textures in images. This approach gives better accuracy results but this takes high computational cost. Usually, texture analysis method depends upon how the texture features are extracted from the image to characterize image. Whenever a new texture feature is derived it is tested whether it is precisely classifies the textures or not. Texture features are most important for precise and accurate texture classification and also important that the way in which they are extracted and applied. The present paper derived a new co-occurrence matrix based on overlapped textons patterns. The present paper generates overlapped texton patterns and generates co-occurrence matrices derived a new matrix called Overlapped Texton Co-occurrence Matrices (OTCOM) for stone texture classification. The present paper integrates the advantages of co-occurrence matrix and texton image by representing the attribute of co-occurrence. The co-occurrence features extracted from the OTCoM provides complete texture information about a texture image. The proposed method is experimented on Vistex, Brodatz textures, CUReT, Mayang, Paul Brooke, and Google color texture images. The experimental results indicate the proposed method classification performance is superior to that of many existing methods.
\end{abstract}

Keywords: co-occurrence matrix, texton, Texture Classification

\section{INTRODUCTION}

Texture classification and segmentation is an important research area from industrial to bio-medical images. The classification problem is basically the problem of identifying an observed textured sample as one of several possible texture classes by a reliable but computationally attractive texture classifier. This implies that the choice of the textural features should be as compact as possible and yet as discriminating as possible. In other words, the extraction of texture features should efficiently embody information about the textural characteristics of the image. The ultimate goal of texture characterization systems is to recognize different textures. To design an effective algorithm for texture classification, it is essential to find a set of texture features with good discriminating power. Previously a number of different texture analysis methods have been introduced namely statistical, structural, transform based and model based methods [1, 2, 3] Normally textures are studied through statistical and syntactical methods. The statistical method measures the coarseness and the directionality of textures in terms of averages on a window of the image $[4,5,6]$. On the other hand syntactical method describes the shape and distribution of the entities. The statistical method has the main features which are to be extracted that includes the autocorrelation function, Fourier transform domain, Markov random field models, local linear transforms, power spectra, difference gray level statistics, co-occurrence matrices and from sum and different statistics $[7,8,9,10,11,12,13]$.
Initially, texture analysis was based on the first order or second order statistics of textures. The co-occurrence matrix features were first proposed by Haralick [6]. Weszka [14] compared texture feature extraction schemes based on the Fourier power spectrum, second order gray level statistics, the co-occurrence statistics and gray level run length statistics. The co occurrence features were found to be the best of these features. This fact is demonstrated in a study by Conners and Harlow [15]. In [16], Haralick features are obtained from wavelet decomposed image yielding improved classification rates.

S.S Sreeja mole [17] in this method classifies the textures on a pixel basis, where each pixel is associated with textural features extracted from co-occurrence matrices that differs the pixel itself. Here the windows related with the adjacent pixels are mostly overlapping resulting the pixels can be obtained by updating values already found. The classification rate in this method is $90 \%$. Jing Yi Tou [18] proposed a method. In this method two popular texture analysis methods i.e. Gabor filters and the Grey Level Cooccurrence Matrices (GLCM). By using this method achieved a recognition rate of $88.52 \%$. Guang-Hai Liu [19] proposed another method. In this method uses the Textons concept and the Grey-level Co-occurrence Matrices (GLCM) techniques used for texture categorization. The preset method uses the combination of the Grey-level Co- 
occurrence Matrices (GLCM) and textons are used for stone texture classification. This method can achieve higher classification rate compare to existing methods. The present paper derived a new co-occurrence matrix based on overlapped textons for texture classification. The new cooccurrence matrix is called as Overlapped Texton Cooccurrence Matrix (OTCoM)

This paper is organized as follows. In Section 2, OTCoM and texture features are proposed. Section 3 discusses results and discussions. Conclusions are given in Section 4.

\section{GENERATION OF OVERLAPPED TEXTON} CO-OCCURRENCE MATRIX (OTCOM) AND

\section{EXTRACTION OF FEATURES}

Various algorithms are proposed by many researchers to extract color, texture and other features. Color is the most distinguishing important and dominant visual feature. That's why color histogram techniques remain popular in the literature. The main drawback of this is, it lacks spatial information. The proposed method consists of three steps which are listed below. In the first step of the proposed method is the color image is converted in to grey level image by using any HSV color model. The following section describes the RGB to HSV conversion procedure

\subsection{RGB to HSV Color Model Conversion}

Recent literature revel various color models in color image processing. In order to extract facial image features from color image information, the proposed method utilized the HSV color space. In the RGB model, images are represented by three components, one for each primary color - red, green and blue. Hue is a color attribute and represents a dominant color. Saturation is an expression of the relative purity or the degree to which a pure color is diluted by white light. HSV color space describes more accurately the perceptual color relationship than RGB color space because it is adopted with a non-linear transform. The present paper has used HSV color space model conversion, because the present study is aimed to classify the human age in to four groups with a gap of 15 years.

HSV color space is created by Hue $(\mathrm{H})$, saturation $(\mathrm{S})$ and value $(\mathrm{V})$. Hue is the property of color such as red, green and blue. Saturation is the intensity of a specific color. Value is brightness of a specific color. However, HSV color space separates the color into three categories i.e. hue, saturation, and value. Separation means variations of color observed individually.

The transformation equations for RGB to HSV color model conversion is given below.

$$
\begin{aligned}
& V=\max (R, G, B) \\
& S=\frac{V-\min (R, G, B)}{V}
\end{aligned}
$$

$$
\begin{gathered}
\mathrm{H}=\frac{\mathrm{G}-\mathrm{B}}{6 \mathrm{~S}} \text { if } \mathrm{V}=\mathrm{R} \\
\mathrm{H}=\frac{1}{3}+\frac{\mathrm{B}-\mathrm{R}}{6 \mathrm{~S}} \text { if } \mathrm{V}=\mathrm{G} \\
\mathrm{H}=\frac{1}{3}+\frac{\mathrm{R}-\mathrm{G}}{6 \mathrm{~S}} \text { if } \mathrm{V}=\mathrm{B}
\end{gathered}
$$

The range of color component Hue $(\mathrm{H})$ is [0,255], the component saturation $(\mathrm{S})$ range is $[0,1]$ and the Value $(\mathrm{V})$ range is $[0,255]$. In this work, the color component Hue $(\mathrm{H})$ is considered as color information for the classification of facial images. Color is an important attribute for image processing applications.

\subsection{Overlapped Texton Matrix Detection}

The texton patterns are defined as a set of blobs or growing patterns sharing a common property on the image [21, 22]. Based on the texton theory, texture can be decomposed into elementary units. Julesz's texton theory mainly focuses on analyzing regular textures, while the overlapped textons can be considered as the extension of Julesz's textons. Since overlapped texton involve texture and shape (edge) information, they can better present features for texture classification.

The present paper utilized a $2 \times 2$ sub window texton pattern as shown in Fig 1(a). In figure 1(a), the four pixel values of a $2 \times 2$ sub window are denoted as $\mathrm{PV}_{1}, \mathrm{PV}_{2}, \mathrm{PV}_{3}$ and $\mathrm{PV}_{4}$. If two pixels are highlighted in gray color of same value then the grid will form a texton. The six texton types denoted as $\mathrm{TP}_{1}, \mathrm{TP}_{2}, \mathrm{TP}_{3}, \mathrm{TP}_{4}, \mathrm{TP}_{5}$ and $\mathrm{TP}_{6}$ are shown in figure $1(\mathrm{~b})$ to $1(\mathrm{~g})$.

\begin{tabular}{|l|l|}
\hline $\mathrm{PV}_{1}$ & $\mathrm{PV}_{2}$ \\
\hline $\mathrm{PV} 3$ & $\mathrm{PV} 4$ \\
\hline
\end{tabular}

(a)

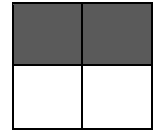

(b)

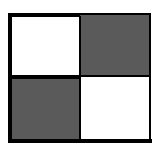

(c)

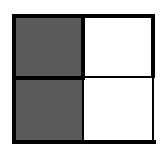

(d)

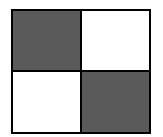

(e)

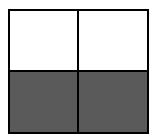

(f)

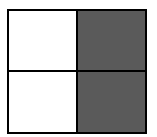

(g)
Fig 1 Six special types of Textons: a) $2 \times 2$ grid b) $\mathrm{TP}_{1}$ c) $\mathrm{TP}_{2}$ d) $\mathrm{TP}_{3}$ e) $\mathrm{TP}_{4}$ f) $\mathrm{TP}_{5}$ and g) $\mathrm{TP}_{6}$.

Julesz's texton theory mainly focuses on analyzing regular textures, but in all those texture analysis use non overlapped texton features for texture analysis. The disadvantage of non overlapped texton pattern, texton matrix consists of more number of zeros so that more information about the image lost. Another major disadvantage is that non overlapped textons does not consider the neighboring texton elements, even though they form the texton pattern with neighboring texton elements. Form Fig. 2 we observe that non overlapped texton image consists more number of zeros. To overcome the above disadvantages overlapped texton concept applies that causes the texton image consists less number of zeros and more information about the image is available for precise texture classification. 


\begin{tabular}{|l|l|l|l|l|l|l|l|}
\hline 151 & 143 & 143 & 143 & 152 & 153 & 146 & 146 \\
\hline 151 & 143 & 143 & 143 & 152 & 153 & 146 & 146 \\
\hline 155 & 142 & 142 & 138 & 147 & 153 & 148 & 148 \\
\hline 157 & 143 & 143 & 135 & 142 & 151 & 149 & 149 \\
\hline 157 & 143 & 143 & 135 & 142 & 151 & 149 & 149 \\
\hline 154 & 146 & 146 & 140 & 143 & 148 & 146 & 146 \\
\hline 154 & 146 & 146 & 140 & 143 & 148 & 146 & 146 \\
\hline 145 & 149 & 149 & 150 & 147 & 144 & 143 & 143 \\
\hline
\end{tabular}

(a)

\begin{tabular}{|c|c|c|c|c|c|c|c|}
\hline 151 & 143 & 143 & 143 & 152 & 153 & 146 & 146 \\
\hline 151 & 143 & 143 & 143 & 152 & 153 & 146 & 146 \\
\hline 0 & 0 & 0 & 0 & 0 & 0 & 148 & 148 \\
\hline 0 & 0 & 0 & 0 & 0 & 0 & 149 & 149 \\
\hline 0 & 0 & 0 & 0 & 0 & 0 & 149 & 149 \\
\hline 0 & 0 & 0 & 0 & 0 & 0 & 146 & 146 \\
\hline 0 & 0 & 0 & 0 & 0 & 0 & 146 & 146 \\
\hline 0 & 0 & 0 & 0 & 0 & 0 & 143 & 143 \\
\hline
\end{tabular}

(b)

Fig 2. Non overlapped texton matrix example (a) original matrix (b) non overlapped matrix

There are many types of texton patterns in texture images. In this paper, we define six types of texton patterns and co-occurrence matrix derived from the overlapped texton pattern image for texture analysis.

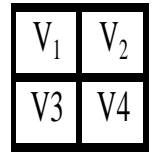

\begin{tabular}{|l|l|l|l|l|l|l|l|}
\hline 151 & 143 & 143 & 143 & 152 & 153 & 146 & 146 \\
\hline 151 & 143 & 143 & 143 & 152 & 153 & 146 & 146 \\
\hline 155 & 142 & 142 & 138 & 147 & 153 & 148 & 148 \\
\hline 157 & 143 & 143 & 135 & 142 & 151 & 149 & 149 \\
\hline 157 & 143 & 143 & 135 & 142 & 151 & 149 & 149 \\
\hline 154 & 146 & 146 & 140 & 143 & 148 & 146 & 146 \\
\hline 154 & 146 & 146 & 140 & 143 & 148 & 146 & 146 \\
\hline 145 & 149 & 149 & 150 & 147 & 144 & 143 & 143 \\
\hline
\end{tabular}

Fig.3: Illustration of the OTM process: (a) $2 \times 2$ grid (b) Original image

\begin{tabular}{|c|c|c|c|c|c|c|c|}
\hline 151 & 143 & 143 & 143 & 152 & 153 & 146 & 146 \\
\hline 151 & 143 & 143 & 143 & 152 & 153 & 146 & 146 \\
\hline 0 & 142 & 142 & 0 & 0 & 153 & 148 & 148 \\
\hline 157 & 143 & 143 & 135 & 142 & 151 & 149 & 149 \\
\hline 157 & 143 & 143 & 135 & 142 & 151 & 149 & 149 \\
\hline 154 & 146 & 146 & 140 & 143 & 148 & 146 & 146 \\
\hline 154 & 146 & 146 & 140 & 143 & 148 & 146 & 146 \\
\hline 0 & 149 & 149 & 0 & 0 & 0 & 143 & 143 \\
\hline
\end{tabular}

(c) Overlapped Texton Matrix (OTM)

\subsection{Co-occurrence Matrix and Features}

Recently Texton Co-occurrence matrix (TCM) is proposed in the literature [27] for higher retrieval rate. TCM is defined to be the distribution of co-occurring texton with a given offset over the texton index image. More over TCM is a computationally expensive procedure. To overcome this, the present paper considers Overlapped Texton Matrix (OTM), which is directly obtained from the original image. To extract precise texture features, the present study computes co occurrence matrix for OTM. Due to cooccurrence matrices are typically large and sparse they are used to measure the texture image. GLCM is proposed by Haralick et al back in 1973 [6]. It is widely used for various texture analysis applications, such as texture Analysis [24], rock texture classification, wood classification and etc. GLCM is a popular statistical technique for extracting textural features from different types of images. In order to find the spatial relationships effectively, the classification method is used and Grey-level co-occurrence matrix (GLCM) is one of the most widely used statistical texture measures. The idea of the method is to consider the relative frequencies for which two neighboring pixels are separated by a distance on the image.
Since the GLCM collects information about pixel pairs instead of single pixels and which is called by a name as second-order statistics.

The GLCM is generated by cumulating the total numbers of grey pixel pairs from the images. Each GLCM will be generated by defining a spatial distance $\mathrm{d}$ and an orientation, which can be 0 degree, 45 degree, 90 degree or 135 degree at a selected grey level G. The GLCM produced will be of size $G \times G$. When the GLCM is constructed, $C_{d}(r, n)$ represents the total pixel pair value where $r$ represents the reference pixel value and $\mathrm{n}$ represents the neighboring pixel value according to the spatial distance and orientation defined. Co occurrence matrix is generated from the OTM is called Overlapped Texton Co-occurrence Matrix (OTCoM). Based on this, OTCoM with different orientations $0^{0}, 45^{\circ}$, $90^{\circ}$, and $135^{\circ}$ are formed as shown in Fig.4(a)-(e) respectively. Textural features are extracted from the OTCoM for classification process. There are a total of fourteen features for GLCM [25]. The textural features used in this method are energy, entropy, contrast, local homogeneity, correlation, and inertia are shown in Eq (1) to Eq (6) [5]. 


\begin{tabular}{|c|c|c|c|c|c|c|c|c|c|c|c|c|c|c|c|}
\hline 3 & 0 & 0 & 0 & 0 & 0 & 0 & 4 & 0 & 0 & 0 & 0 & 0 & 0 & 0 & 7 \\
\hline 0 & 0 & 0 & 0 & 0 & 0 & 0 & 0 & 0 & 0 & 0 & 0 & 0 & 0 & 0 & 0 \\
\hline 0 & 0 & 0 & 0 & 0 & 0 & 0 & 0 & 0 & 0 & 0 & 0 & 0 & 0 & 0 & 0 \\
\hline 0 & 0 & 0 & 0 & 0 & 0 & 0 & 0 & 0 & 0 & 0 & 0 & 0 & 0 & 0 & 0 \\
\hline 0 & 0 & 0 & 0 & 0 & 0 & 0 & 0 & 0 & 0 & 0 & 0 & 0 & 0 & 0 & 0 \\
\hline 0 & 0 & 0 & 0 & 0 & 0 & 0 & 0 & 0 & 0 & 0 & 0 & 0 & 0 & 0 & 0 \\
\hline 0 & 0 & 0 & 0 & 0 & 0 & 0 & 0 & 0 & 0 & 0 & 0 & 0 & 0 & 0 & 0 \\
\hline 2 & 0 & 0 & 0 & 0 & 0 & 0 & 47 & 3 & 0 & 0 & 0 & 0 & 0 & 0 & 46 \\
\hline \multicolumn{8}{|c|}{$/ / / / / / /(\mathrm{a})$} & \multicolumn{8}{|c|}{ (b) } \\
\hline 0 & 0 & 0 & 0 & 0 & 0 & 0 & 7 & 0 & 0 & 0 & 0 & 0 & 0 & 0 & 5 \\
\hline 0 & 0 & 0 & 0 & 0 & 0 & 0 & 0 & 0 & 0 & 0 & 0 & 0 & 0 & 0 & 0 \\
\hline 0 & 0 & 0 & 0 & 0 & 0 & 0 & 0 & 0 & 0 & 0 & 0 & 0 & 0 & 0 & 0 \\
\hline 0 & 0 & 0 & 0 & 0 & 0 & 0 & 0 & 0 & 0 & 0 & 0 & 0 & 0 & 0 & 0 \\
\hline 0 & 0 & 0 & 0 & 0 & 0 & 0 & 0 & 0 & 0 & 0 & 0 & 0 & 0 & 0 & 0 \\
\hline 0 & 0 & 0 & 0 & 0 & 0 & 0 & 0 & 0 & 0 & 0 & 0 & 0 & 0 & 0 & 0 \\
\hline 0 & 0 & 0 & 0 & 0 & 0 & 0 & 0 & 0 & 0 & 0 & 0 & 0 & 0 & 0 & 0 \\
\hline 2 & 0 & 0 & 0 & 0 & 0 & 0 & 40 & 3 & 0 & 0 & 0 & 0 & 0 & 0 & 41 \\
\hline \multicolumn{8}{|c|}{ (c) } & \multicolumn{8}{|c|}{ (d) } \\
\hline
\end{tabular}

Fig. 4: a) Overlapped Texton matrix (b) (c), (d) and (e) represents the co occurrences on OTCoM of $0^{0}, 45^{\circ}, 90^{\circ}$ and $135^{\circ}$.

$$
\begin{aligned}
& \text { Entropy }=\sum_{i, j=0}^{N-1}-\ln \left(P_{i j}\right) P_{i j} \\
& \text { Energy }=\sum_{i, j=0}^{N-1}-\ln \left(P_{i j}\right)^{2} \\
& \text { Contrast }=\sum_{i, j=0}^{N-1} P_{i j}(i-j)^{2}
\end{aligned}
$$

\section{RESULTS AND DISCUSSIONS}

The present paper carried out the experiments on two Datasets. The Dataset-1 consists of various Mosaic, Granite, Marble and Brick stone textures with resolution of $256 \times 256$ collected Vistex, Brodatz textures, Mayang, Google color texture images and also from natural images taken by using digital camera. Some of stone texture images in Dataset-1 are shown in the Fig. 5. The Dataset-2 consists of various Mosaic, Granite, Marble and Brick stone textures with resolution of $256 \times 256$ collected from CUReT, Paul Bourke, and also from natural images taken by using digital camera. Some of images in Dataset2 are shown in the Fig. 6. Dataset- 1 and Dataset- 2 contains 80 and 96 original color texture images respectively.

Every texture image is subdivided into 16 sub images of non-overlapped image regions of size $(64 \times 64)$. This results into a total of 2816 sub image regions. The classification is done for all sub texture regions derived from each texture image in Dataset-1 and Dataset-2. Feature set leads to representation of the training and testing textures features. The absolute difference of the feature vector values of the query texture and database textures are also calculated.

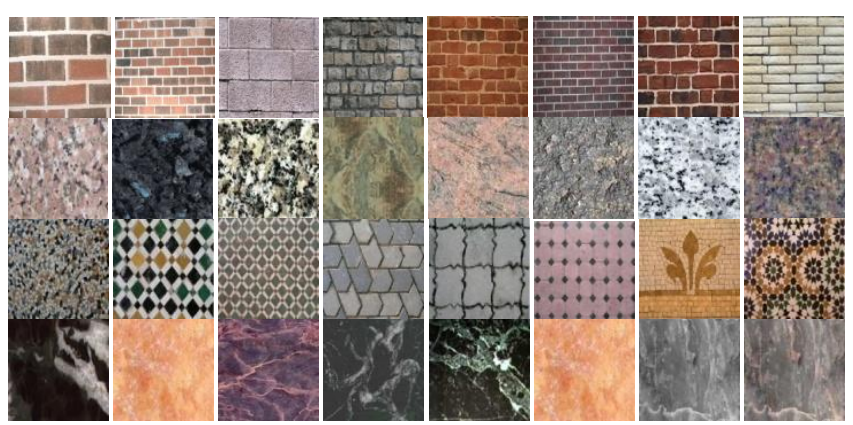

Fig.5: Input texture group of 8 samples of Brick, Granite, Mosaic., Marble with size of $256 \times 256$ 


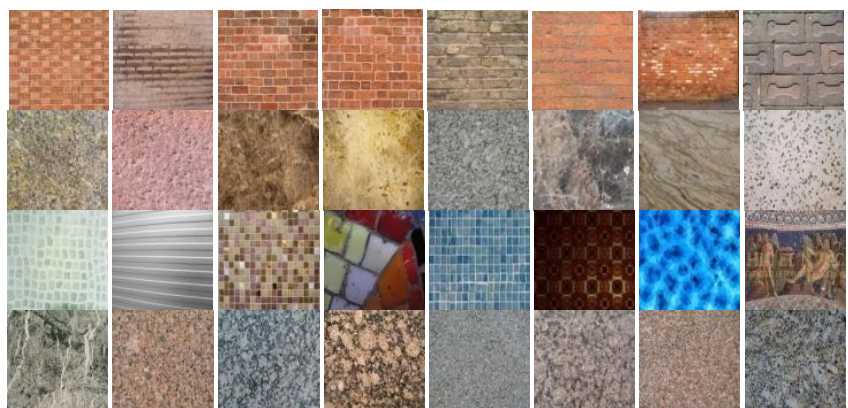

Fig 6: Input texture group of 8 samples of Brick, Granite, Mosaic, Marble with size of $200 \times 150$

To classify the relevant textures, fixed threshold, $K-N N$ classifier is used to measure the similarity between query texture and the database textures. In case of fixed threshold, the threshold values are computed for different query textures. The best threshold value is chosen as the threshold of that particular texture feature. The Euclidean distance between these FVs helps in classifying the texture into correct group.

The results from two datasets are obtained in Table $1 \& 2$ which shows the average classification rates of the proposed OTCoM method.

Table 1a: Database-1: (\%) mean classification rate of brick and marble stone textures

\begin{tabular}{|l|l|l|l|l|}
\hline Sno & Texture Name & Classification Rate & Texture Name & Classification Rate \\
\hline 1 & Brick1 & 96.75 & marble1 & 95.45 \\
\hline 2 & Brick2 & 92.81 & marble2 & 97.47 \\
\hline 3 & Brick3 & 90.34 & marble3 & 95.12 \\
\hline 4 & Brick4 & 96.28 & marble4 & 96.58 \\
\hline 5 & Brick5 & 97.47 & marble5 & 91.78 \\
\hline 6 & Brick6 & 96.9 & marble6 & 87.57 \\
\hline 7 & Brick7 & 90.92 & marble7 & 93.65 \\
\hline 8 & Brick8 & 92.71 & marble8 & 93.78 \\
\hline 9 & Brick9 & 91.29 & marble9 & 97.42 \\
\hline 10 & Brick10 & 96.62 & marble10 & 87.53 \\
\hline 11 & Brick11 & 94.74 & marble11 & 95.86 \\
\hline 12 & Brick12 & 93.17 & marble12 & 91.79 \\
\hline 13 & Brick13 & 91.71 & marble13 & 95.89 \\
\hline 14 & Brick14 & 92.76 & marble14 & 95.67 \\
\hline 15 & Brick15 & 91.76 & marble15 & 91.17 \\
\hline 16 & Brick16 & 93.37 & marble16 & 96.37 \\
\hline 17 & Brick17 & 91.76 & marble17 & 90.22 \\
\hline 18 & Brick18 & 91.79 & marble18 & 96.23 \\
\hline 19 & Brick19 & 91.71 & marble19 & 95.53 \\
\hline 20 & Brick20 & 91.76 & marble20 & 91.75 \\
\hline Average & & $\mathbf{9 3 . 3 3 1}$ & Average & $\mathbf{9 3 . 8 4 1 5}$ \\
\hline
\end{tabular}

Table 1b: Database-1: (\%) mean classification rate of mosaic and granite stone textures

\begin{tabular}{|l|l|l|l|l|}
\hline Sno & Texture Name & Classification Rate & Texture Name & Classification Rate \\
\hline 1 & granite1 & 91.51 & mosiac1 & 93.87 \\
\hline 2 & granite2 & 91.72 & mosiac2 & 90.38 \\
\hline 3 & granite3 & 99.68 & mosiac3 & 97.33 \\
\hline 4 & granite4 & 87.56 & mosiac4 & 91.76 \\
\hline 5 & granite5 & 90.81 & mosiac5 & 89.73 \\
\hline 6 & granite6 & 95.56 & mosiac6 & 97.19 \\
\hline 7 & granite7 & 79.29 & mosiac7 & 83.37 \\
\hline 8 & granite8 & 83.34 & mosiac8 & 91.77 \\
\hline 9 & granite9 & 90.53 & mosiac9 & 95.98 \\
\hline 10 & granite10 & 96.27 & mosiac10 & 94.71 \\
\hline 11 & granite11 & 91.72 & mosiac11 & 98.24 \\
\hline
\end{tabular}




\begin{tabular}{|l|l|l|l|l|}
12 & granite12 & 99.74 & mosiac12 & 96.91 \\
\hline 13 & granite13 & 97.62 & mosiac13 & 97.52 \\
\hline 14 & granite14 & 91.75 & mosiac14 & 92.74 \\
\hline 15 & granite15 & 96.93 & mosiac15 & 97.63 \\
\hline 16 & granite16 & 97.19 & mosiac16 & 97.48 \\
\hline 17 & granite17 & 98.23 & mosiac17 & 91.91 \\
\hline 18 & granite18 & 96.83 & mosiac18 & 97.24 \\
\hline 19 & granite19 & 91.85 & mosiac19 & 91.56 \\
\hline 20 & granite20 & 91.78 & mosiac20 & 97.64 \\
\hline Average & & $\mathbf{9 4 . 2 4 8}$ & Average & $\mathbf{9 4 . 2 4 8}$ \\
\hline
\end{tabular}

Table 2a: Database-2: (\%) mean classification rate of brick and marble stone textures

\begin{tabular}{|c|c|c|c|c|}
\hline Sno & Texture Name & Classification Rate & Texture Name & Classification Rate \\
\hline 1 & Brick1 & 92.90 & marble1 & 93.00 \\
\hline 2 & Brick2 & 98.13 & marble2 & 91.7 \\
\hline 3 & Brick3 & 93.63 & marble3 & 91.65 \\
\hline 4 & Brick4 & 95.37 & marble4 & 94.17 \\
\hline 5 & Brick5 & 93 & marble5 & 94.13 \\
\hline 6 & Brick6 & 93.57 & marble6 & 89.53 \\
\hline 7 & Brick7 & 96.13 & marble7 & 97.53 \\
\hline 8 & Brick8 & 94.77 & marble8 & 88.37 \\
\hline 9 & Brick9 & 93.07 & marble9 & 95.37 \\
\hline 10 & Brick10 & 93.97 & marble10 & 94.6 \\
\hline 11 & Brick11 & 93.75 & marble11 & 87.5 \\
\hline 12 & Brick12 & 97.13 & marble12 & 98.03 \\
\hline 13 & Brick13 & 91.25 & marble13 & 88.37 \\
\hline 14 & Brick14 & 88.37 & marble14 & 99.83 \\
\hline 15 & Brick15 & 88.37 & marble15 & 96.43 \\
\hline 16 & Brick16 & 88.37 & marble16 & 97.43 \\
\hline 17 & Brick17 & 88.37 & marble17 & 88.37 \\
\hline 18 & Brick18 & 94.37 & marble18 & 92.70 \\
\hline 19 & Brick19 & 97.67 & marble19 & 88.37 \\
\hline 20 & Brick20 & 89.5 & marble20 & 89.6 \\
\hline 21 & Brick21 & 93.50 & marble21 & 91.75 \\
\hline 22 & Brick22 & 91.90 & marble22 & 95.85 \\
\hline 23 & Brick23 & 95.20 & marble23 & 92.03 \\
\hline 24 & Brick24 & 91.13 & marble24 & 95.70 \\
\hline \multicolumn{2}{|c|}{ Average } & 93.06 & Average & 93.00 \\
\hline
\end{tabular}

Table 2a: Database-2: (\%) mean classification rate of granite and marble stone textures

\begin{tabular}{|l|l|l|l|l|}
\hline Sno & Texture Name & Classification Rate & Texture Name & Classification Rate \\
\hline 1 & granite1 & & mosiac1 & 94.3 \\
\hline 2 & granite2 & 93.75 & mosiac2 & 93.53 \\
\hline 3 & granite3 & 91.6 & mosiac3 & 88.37 \\
\hline 4 & granite4 & 94.97 & mosiac4 & 93.50 \\
\hline 5 & granite5 & 88.37 & mosiac5 & 91.45 \\
\hline 6 & granite6 & 88.37 & mosiac6 & 90.80 \\
\hline 7 & granite7 & 93.43 & mosiac7 & 96.83 \\
\hline 8 & granite8 & 88.37 & mosiac8 & 90.17 \\
\hline 9 & granite9 & 96.67 & mosiac9 & 95.53 \\
\hline 10 & granite10 & 93.07 & mosiac10 & 90.20 \\
\hline 11 & granite11 & 94.55 & mosiac11 & 88.37 \\
\hline 12 & granite12 & 93.07 & mosiac12 & 85.45 \\
\hline 13 & granite13 & 95.33 & mosiac13 & 95.23 \\
\hline 14 & granite14 & 94.83 & mosiac14 & 93.37 \\
\hline 15 & granite15 & 90.73 & mosiac15 & 95.23 \\
\hline 16 & granite16 & 93.57 & mosiac16 & 91.7 \\
\hline 17 & granite17 & 93.40 & mosiac17 & 91.7 \\
\hline
\end{tabular}




\begin{tabular}{|l|l|l|l|l|}
18 & granite18 & 90.60 & mosiac18 & 94.43 \\
\hline 19 & granite19 & 89.6 & mosiac19 & 91.7 \\
\hline 20 & granite20 & 92.17 & mosiac20 & 96.65 \\
\hline 21 & granite21 & 98.83 & mosiac21 & 97.33 \\
\hline 22 & granite22 & 91.70 & mosiac22 & 98.13 \\
\hline 23 & granite23 & 92.43 & mosiac23 & 97.43 \\
\hline 24 & granite24 & 96.20 & mosiac24 & 97.33 \\
\hline Average & $\mathbf{9 2 . 8 5}$ & Average & $\mathbf{9 3 . 2 8}$ \\
\hline
\end{tabular}

Mean classification rates for the proposed OTCoM and the other existing methods for classification stone textures using K-NN classifier is shown in Table 3 which clearly indicates that the proposed OTCoM outperforms the other existing methods. Fig.7 shows the comparison chart of the existing methods which are specified in table 3 and proposed OTCoM method.

Table 3: Mean classification rates for the two different texture image datasets using k-NN classifier

\begin{tabular}{|l|l|l|l|}
\hline Image Dataset & Pixel Based image Classification & GLCM and Gabor Filters & Proposed Method FBTCoM \\
\hline Brodatz & 90.34 & 88.53 & 96.93 \\
\hline VisTex & 89.12 & 88.15 & 97.19 \\
\hline Mayang & 91.23 & 87.08 & 98.23 \\
\hline CUReT & 89.12 & 84.61 & 96.83 \\
\hline Paul Bourke & 91.23 & 87.01 & 94.55 \\
\hline
\end{tabular}

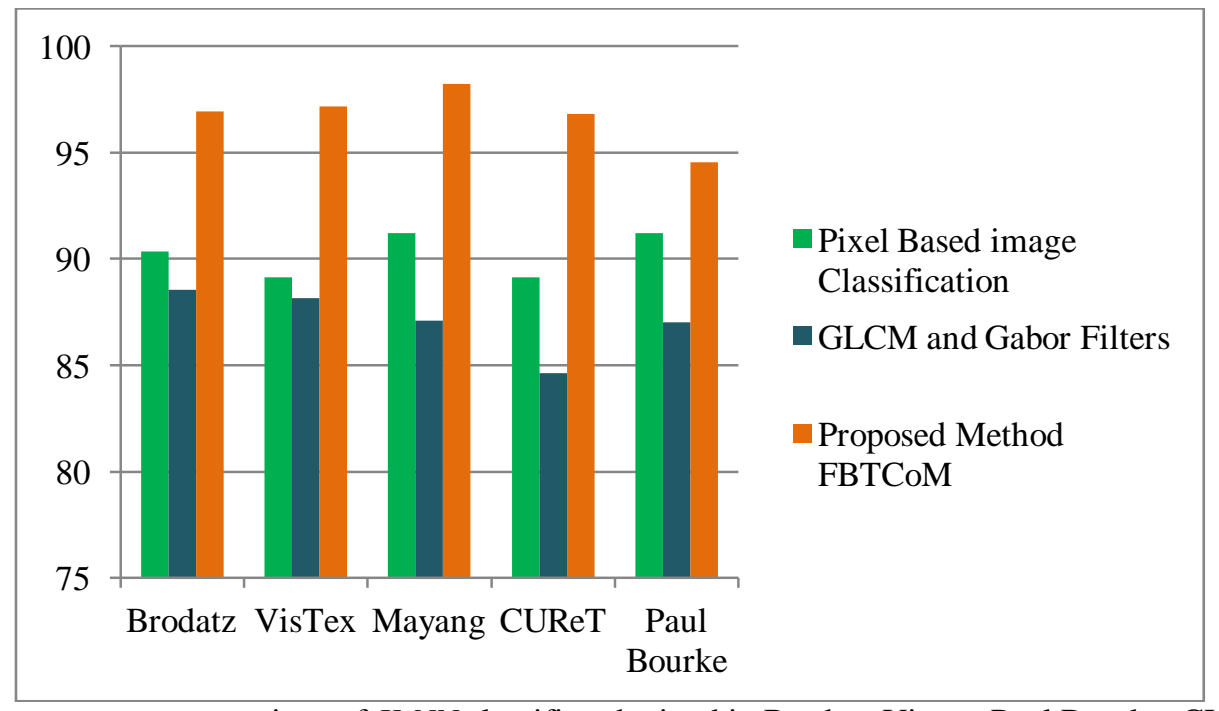

Fig 7: Classification accuracy comparison of $K-N N$ classifier obtained in Brodatz Vistex, Paul Bourke, CUReT, and Mayang dataset for Pixel Based image Classification, GLCM and Gabor Filters and proposed method.

\section{CONCLUSION}

Paragraph The present paper derived a new co-occurrence matrix called as Overlapped Texton Co-occurrence Matrix (OTCoM) for rotation invariant texture classification. Julesz [21] proposed texton which represents the patterns of texture which is useful in texture analysis. The disadvantage of TCM is that, the computationally expensive. To overcome this problem, the present paper considered overlapped Texton Matrix (OTM), which is directly obtained from a original image and to extract a precise texture features.

The features on color textures are extracted by means of GLCM [17] statistical method though the concept of overlapping window for neighboring pixels. So that, it is the computationally expensive. The present paper statistical method though the concept of non overlapping window for neighboring pixels. The experimental results clearly indicate the efficacy of the proposed OTCoM over the various existing methods.

\section{REFERENCES}

[1] "Measuring Texture Classification Algorithms" by G. Smith and I. Burns, , Pattern Recognition Letters, 1997, vol. 18, pp. 1495-1501

[2] "Statistical and Structural Approaches to Texture," by Haralick, R.M., Proceedings of the IEEE, 67, pp. 786-804, 1979

[ 3 ] 'A theoretical comparison of texture algorithms' by Conners, r.w., and harlow, C.A.:, IEEE Transactions., May 1980, PAMI-2, pp. 20.5222

[4] Texture Classification By Using Advanced Local Binary Patterns And Spatial Distribution Of Dominant Patterns", by Shu Liao and Albert C. S. Chung “in 2007.pp.1221-1224

[ 5 ] "A comparative study of texture measures for terrain classification" by Weszka, j., dyer, c., and rosenfeld, A in IEEE Transactions. April 1976, SMC- 6, (4), pp. 269-285 
[ 6 ] Haralick, r.m., shanmugam, k., and dinstein, “Textural features for image Classification', IEEE Transactions, November 1973, SMCJ, (6), pp. 610621

[7] Unser, M.: 'Sum and difference histograms for texture classification', IEEE Trans., January 1986, PAMI-8, (I), pp. 118-125

[ 8 ] Davis, 1.s., johns, s.a., and aggarwal, J.K.: "Texture analysis using generalized co-occurrence matrices', IEEE Trans.,July 1979, PAMI-1, (3). pp. 251-259

[9] Alparone, 1., argenti, f., and benelli, g.: 'Fast calculation of co-occurrence matrix parameters for image segmentation',Electron. Lett.,January 1990,26, (I), pp. 23-24

[10] A. Laine and J. Fan, "Texture classification by wavelet packet signatures", IEEE Transactions. on PAMI, 15(11), 1993, pp. 1186-1190.

[11] "Multichannel Texture Analysis Using Localized Spatial Filters" by A. Bovik, M. Clark, W. S. Geisler, IEEE Transactions on Pattern Analysis and Machine Intelligence, 12 (1), 1990, pp. 55-73.

[12] A. K. Jain and F. Farrokhnia, "Unsupervised texture segmentation using Gabor filters", in Pattern Recognition, 24(12), 1991, pp. 1167-1186.

[ 13 ] M. Unser, M. Eden, "Multiresolution feature extraction and selection for texture segmentation", IEEE Transactions on Pattern Analysis and Machine Intelligence, 11, 1989, pp. 717-728

[14] J. S. Weszka, C. R. Dyer, A. Rosenfeld, "A comparative study of texture measures for terrain classification", IEEE Transactions in System Man Cybernat,6(4), 1976, pp. 269-285.

[ 15 ] R. W. Conners and C. A. Harlow. "A Theoretical Comparison Texture Algorithms", IEEE Transactions on Pattern Analysis and Machine Intelligence, 2, 1980, pp. 204-222.

[ 16] "Texture classification using wavelet transform" by S.Arivazhagan, L. Ganesan, , Pattern Recognition Letters, 24, 2003, pp. 1513-1521.

[17] "Texture classification using Wavelet Packet Decomposition", by P. S.Hiremath, and S. Shivashankar, ICGSTs GVIP Journal, 6(2), 2006, pp. 77-80.

[18] "Pixel Based Classification On Color Images In Statistical Texture Analysis" by S.S Sreeja Mole, Dr.L.Ganesan, IJCSET -2010, vol 1 no 2 pages 4146.

[19] "Gabor Filters and Grey-level Co-occurrence Matrices in Texture Classification" by Jing Yi Tou, Yong Haur Tay and Phooi Yee Lau Pattern Recognition 34, 2001, pp. 727-739.

[20] Julesz B., - Textons, The Elements of Texture Perception, and their Interac-tions," Nature, vol.290 (5802): pp.91-97, 1981.

[21] Julesz B., -Texton gradients: the texton theory revisited," Biological Cybernet-ics, vol.54 pp.245251, 1986.

[ 22 ] Guang-Hai Liu, Zuo-Yong Li, Lei Zhang, Yong Xu, "Image retrieval based on micro-structure descriptor," Pattern Recognition, vol. 44, pp:21232133, 2011.

[23 ] M. Tuceryan, and A. K. Jain, "Texture Analysis, The Handbook of Pattern Recognition and Computer Vision, Ed. 2", World Scientific Publishing Co., 1998.

[24] "Design of an Intelligent Wood Recognition System for the Classification of Tropical Wood Species", by Y. L. Lew, Master of Engineering (Electrical) thesis, Faculty of Electrical Engineering, Universiti Teknologi Malaysia, Malaysia, 2005

[ 25 ] M. Petrou, and P. G. Sevilla, "Image Processing: Dealing with Texture", Wiley, 2006

[26] N. Jhanwar, S. Chaudhuri, G. Seetharaman, B. Zavidovique "Content based image retrieval using motif cooccurrence matrix", Image and Vision Computing 22 (2004) 1211-1220

[27] Guang-Hai Liu*, Jing-Yu Yang "Image retrieval based on the texton co-occurrence matrix", Pattern Recognition 41 (2008) 3521 -- 3527

\section{BIOGRAPHIES}

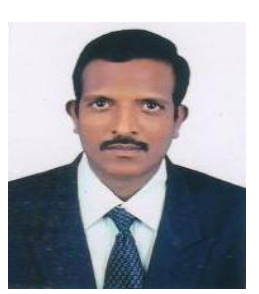

Dr. Patnala S. R. Chandra Murty completed B.Tech in the year of 2005 from JNTu hydrabad and M,Tech from ANU, Guntur in the year of 2008 . He received $\mathrm{Ph} \mathrm{D}$ degree in Computer Science and Engineering from JNTUK, Kakinada in year 2013. He published 8 papers in National and International Journals and 7 papers in National and International Conferences. Mail Id chandra_psr@rediffmail.com

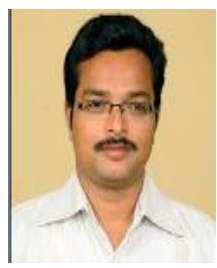

Dr. U Ravi Babu completed M,Tech from RVD university in the year of 2005 . He received $\mathrm{Ph} \mathrm{D}$ degree in Computer Science and Engineering from ANU, Guntur in year 2013. He published 10 papers in National and International Journals and 9 papers in National and International Conferences. He working as professor in the depart of CSE at Malla Reddy Engendering College (Autonomous). He is life member of ISCA, IRS, IAENG and CSTA. Mail id: uppu.ravibabu@gmail.com

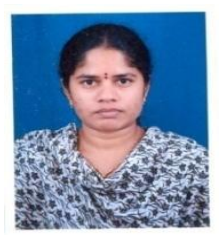

R. Venkata Lakshmi received B.Tech degree in Computer Science \& Engineering from Acharya Nagarjuna University. She received her M.Tech degree in Computer Science \& Engineering from Andhra University. Presently she is doing her Ph.D in JNTU kainada. Her Area of interest is Image Processing. 